Hi gh- Tc SQUl D magnet omet er s for use i n moder at emagnet i cal I y- shi el ded room

\begin{tabular}{|c|c|}
\hline 著者 & $\begin{array}{l}\text { MATSUDA M zushi, ONO S., KATO K, MATSUURA } \\
\begin{array}{lll}\text { T. , OYAMA H., HAYASH } & \text { A., H RANO S., KURI K } \\
\text { S., YOKOSAMA K } & & \end{array}\end{array}$ \\
\hline $\begin{array}{l}\text { j our nal or } \\
\text { publ i cat i on titl e }\end{array}$ & I EEE transacti ons on appl i ed super conduct i vi ty \\
\hline vol une & 11 \\
\hline nunber & 1 \\
\hline page $r$ ange & $1323-1326$ \\
\hline year & 2001- 03 \\
\hline URL & ht t p: //hdl . handl e. net /10258/206 \\
\hline
\end{tabular}




\section{High-Tc SQUID Magnetometers for Use in Moderate Magnetically-Shielded Room}

M. Matsuda, S. Ono, K. Kato, T. Matsuura, H. Oyama, A. Hayashi, S. Hirano, S. Kuriki, and K. Yokosawa

\begin{abstract}
We have fabricated and characterized high- $T_{c}$ planar SQUID magnetometers and first derivative gradiometers with directly-coupled pickup loops. The devices were made from single layer of $\mathrm{YBa}_{2} \mathrm{Cu}_{3} \mathrm{O}_{7-\delta}$ thin film on LSAT bicrystal substrate with $30^{\circ}$ or $24^{\circ}$ misorientation angle.

Magnetic properties were investigated by applying a magnetic field $B_{0}$ for the SQUID magnetometer patterned with holes to reduce the maximum structural width. We found an increasing low frequency noise with cooling fields $B_{\theta}$ larger than $1.5 \mu \mathrm{T}$. This value consists with the threshold field estimated from the maximum structural width. A magnetic field noise level of $500 \mathrm{fT} / \mathrm{Hz}^{1 / 2}$ at $10 \mathrm{~Hz}$ was observed by using $\mathrm{FLL}$ electronics with a bias current reversal in a moderate magnetically-shielded room consisting of only two $1 \mathrm{~mm}$ thick layers of permalloy. Measurements of magnetocardiograms demonstrate the suitability of this magnetometer for biomagnetic applications.

On the other hand, the gradiometer with two symmetric pickup loops was operated without any shielding. The performance obtained was a field gradient resolution of about 1 $\mathrm{pT} / \mathrm{cmHz}^{1 / 2}$ at $1 \mathrm{kHz}$ and $10 \mathrm{pT} / \mathrm{cmHz}^{1 / 2}$ at $1 \mathrm{~Hz}$, with a baseline of $4 \mathrm{~mm}$. The imbalance of this gradiometer was around $0.7 \%$, limited by the sensitivity to homogeneous field of the SQUID itself.
\end{abstract}

Index Terms-SQUIDs, noise properties, magnetometer, first derivative gradiometer, magnetocardiogram

\section{INTRODUCTION}

$\mathrm{T}$ HERE is a great interest in using high- $T_{c}$ dc superconducting quantum interference devices (SQUIDs) for biomagnetic applications such as measurements of the magnetocardiogram (MCG). For practical use of the SQUID, it is desirable to work in unshielded environment, or in very simple and less expensive magnetically-shielded rooms (MSR) because of the enormous cost reduction. It is necessary to have low noise for the SQUID at low frequencies, where most biomagnetic signals are included, even in such a moderate MSR. In general, SQUIDs suffer

Manuscript received September 18, 2000. This work was partially supported by a Grants-in-Aid for Scientific Research (No. 10142101 and 11359001) from the Ministry of Education, Science, Sports, and Culture, Japan..

M. Matsuda, S. Ono, K. Kato, and T. Matsuura are with Muroran Institute of Technology, Muroran, Hokkaido, JAPAN (telephone: +81-143-46-5550, e-mail: matsuda @mmm.muroran-it.ac.jp).

S. Kuriki, H. Oyama, A. Hayashi, and S. Hirano are with Research Institute for Electronic Science, Hokkaido University, Sapporo, Hokkaido, JAPAN (e-mail: sk@es.hokudai.ac.jp).

K. Yokosawa is with Central Research Laboratory, Hitachi Ltd., Kokubunji, Tokyo, JAPAN (e-mail: yokosawa@crl.hitachi.co.jp). from increased low frequency noise when operated in a relatively large residual magnetic field. This has been explained in the framework of thermally activated hopping of magnetic flux vortices trapped at local pinning sites in the superconducting film of the SQUID body [1]. It has been reported that flux entry into the superconductor has been avoided by reduction of the superconducting line width [2], [3]. An estimation for the threshold field of flux entry is given by $B_{T}=\left(\pi \Phi_{0}\right) /\left(4 w^{2}\right)$, where $w$ is the linewidth of the film. Here, we describe the magnetic properties in static field for our SQUID magnetometer, patterned with holes to reduce the maximum structural width of the pickup loop. The performance of the magnetometer is demonstrated by measuring the MCG signals inside a moderate MRS.

Another way to reduce the effect of residual field noise in a poor-shielded environment is to form a gradiometer configuration. The biomagnetic signal is very localized compared with other environmental disturbances like the earth's magnetic field. Gradiometers which are sensitive only to the field gradient and suppress homogeneous background fields, are much advantageous over magnetometers. Thus, recently directly coupled gradiometers made from single $\mathrm{YBa}_{2} \mathrm{Cu}_{3} \mathrm{O}_{7-\delta}(\mathrm{YBCO})$ layers attracts more attention [4]-[6]. In this work, we have measured characteristics of the SQUID gradiometer under different field environments. Operation of flux dams included within the pickup loops was also confirmed.

\section{MAGNETOMETER WITH HOLES}

\section{A. Design and Fabrication}

The SQUID magnetometers are prepared from c-axis oriented YBCO films. A $250 \mathrm{~nm}$ thick film of YBCO and a $200 \mathrm{~nm}$ thick of $\mathrm{Au}$ was deposited by a co-evaporation method on a $10 \mathrm{~mm} \times 10 \mathrm{~mm}\left[\mathrm{LaAlO}_{3}\right]_{0.3}\left[\mathrm{Sr}(\mathrm{Al}, \mathrm{Ta}) \mathrm{O}_{3}\right]_{0.7}$ (LSAT) bicrystal substrate having low permittivity with a misorientation angle of $30^{\circ}$. The films were patterned by Ar ion beam etching and standard photolithography technique into the geometry illustrated in Fig. 1. The transverse-type SQUID has two parallel strips of $5 \mu \mathrm{m}$ width and a long hole parallel to the bicrystal line. The hole length and width are 60 and $10 \mu \mathrm{m}$, respectively. The $2 \mu \mathrm{m}$ wide bridges including grain boundary junctions were located at the both side of the hole. The inductance value estimated using the coplanar stripline formula which includes the kinetic inductance is 


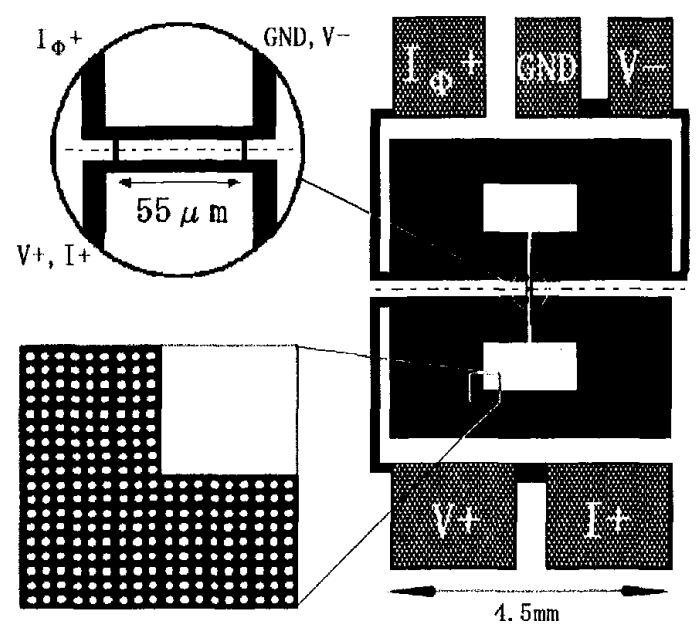

Fig. 1. Layout of a complete magnetometer and the inner part of the sensor around the SQUID (The dashed line indicates the grain boundary).

about $70 \mathrm{pH}$. We formed many holes $(15 \mu \mathrm{m} \phi)$ in the pickup loop to divide the YBCO film into a maximum structural width of $35 \mu \mathrm{m}$, corresponding to $1.5 \mu \mathrm{T}$ of $B_{T}$. The measured effective area of this magnetometer was about $0.12 \mathrm{~mm}^{2}$.

\section{B. Magnetic Properties of Magnetometer}

The characterization and noise measurements were performed inside a moderate MSR consisting of only two $1 \mathrm{~mm}$ thick layers of permalloy. The static magnetic field, generated by a solenoid coil was applied to the device perpendicular to the substrate. In field-cooled case, we immersed the device into liquid nitrogen slowly in the

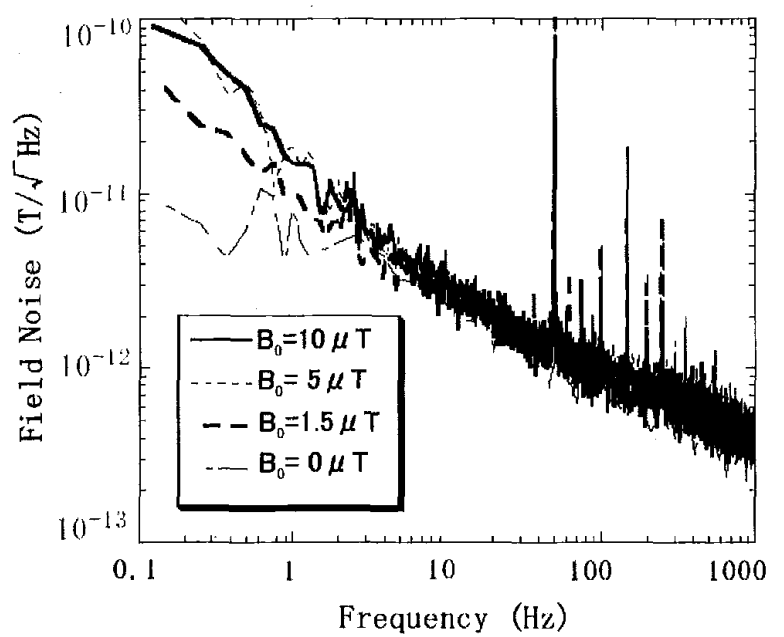

Fig. 2. Flux density noise spectra of the magnetometer for different cooling fields $B_{0}$.

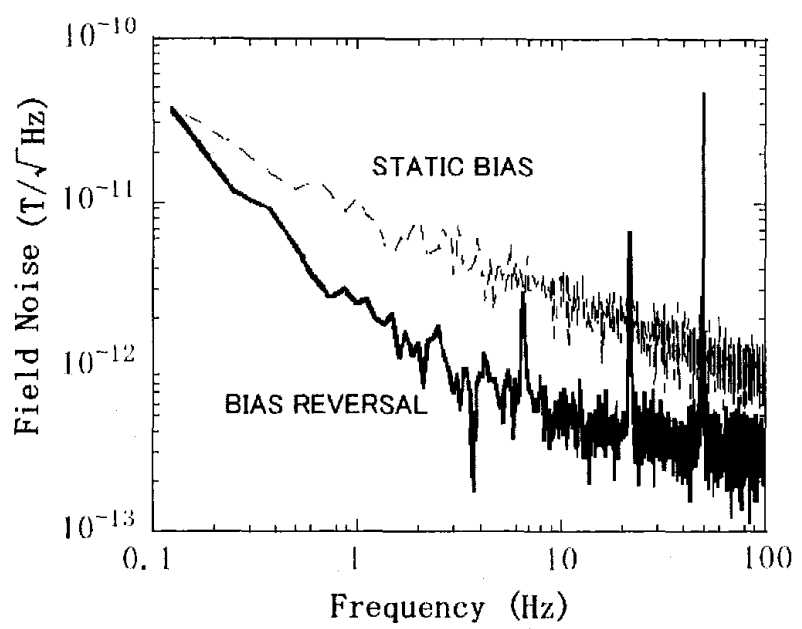

Fig. 3. Magnetic field noise of the magnetometer in a moderate MSR using FLL electronics.

presence of static magnetic field. Fig. 2 shows several flux density noise spectra, in FLL operation of static bias current, for the magnetometer in different cooling fields $B_{0}$. We find an increasing low frequency noise with $B_{0}$ larger than $1.5 \mu \mathrm{T}$. This value consists with the threshold field estimated from the maximum structural width.

On the other hand, in zero field-cooled case, we applied the field at $77 \mathrm{~K}$ after superconductive transition of the device. In this case, a critical current of the SQUID has Fraunhofer-like dependence on $B_{0}$, i.e., on the magnetic field generated by shielding currents flowing the SQUID loop. For larger $B_{0}$ than $15 \mu \mathrm{T}$, the vortices seem to be introduced into the film by the Lorentz force from shielding currents.

For practical applications, we used flux-locked loop (FLL) electronics using $25 \mathrm{kHz}$ flux modulation with $200 \mathrm{kHz}$ bias current reversal scheme to reject the low frequency noise from parameter fluctuations in the junctions. Fig. 3 shows the magnetic field noise spectra of the magnetometer in a moderate MSR. For comparison, the result for static bias is also shown in this figure. A field noise level of $500 \mathrm{fT} / \mathrm{Hz}^{1 / 2}$ at $10 \mathrm{~Hz}$ was observed by using a bias current reversal.

\section{MCG Measurement in a moderate MSR}

In order to demonstrate the noise performance of the magnetometer, MCG signals were recorded at a position of approximately maximum peak intensity inside a moderate MSR. The distance between the magnetometer and the chest surface was about $30 \mathrm{~mm}$. The measurement bandwidth was 1 to $40 \mathrm{~Hz}$. Fig. 4 shows the real-time traces and the spectra of the MCG signal (a) and background signal (b) from a male subject. Here, the magnetometer is just above the abdominal region for (b). QRS-complex peaks of about 50 $\mathrm{pT}_{\mathrm{pp}}$ are clearly observed, and a $20 \mathrm{~Hz}$ component originating from the vibration of the MSR is dominant in the noise levels of $5 \mathrm{pT}_{\mathrm{pp}}$. 


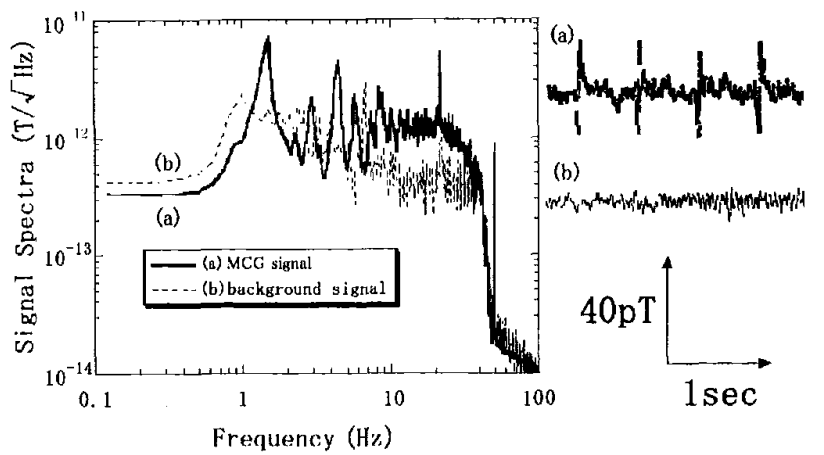

Fig. 4. Real-time trace and the spectra of the MCG signal (a) and background signal (b) from a male subject (Bandwidth: 1 to $40 \mathrm{~Hz}$ ).

\section{FIRST-ORDER GRDIOMETER}

\section{A. Design}

The SQUID gradiometer was prepared by the same procedure as the magnetometer. An YBCO film of $200 \mathrm{~nm}$ on LSAT bicrystal substrate having a misorientation angle of $24^{\circ}$ was used. As shown in Fig. 5, it consists of two symmetric and approximately rectangular pickup loops to which the longitudinal-type SQUID is directly coupled. In this geometry, high flux coupling coefficient $\alpha$ of around 0.8 was obtained [7]. The estimated inductance $L_{p}$ and an effective area $A_{p}$ for one pickup loop are $4 \mathrm{nH}$ and $10 \mathrm{~mm}^{2}$, respectively. The baseline $2 b$ is $4 \mathrm{~mm}$. This device includes flux dams with 50 $\mu \mathrm{m}$ width within the pickup loop to suppress the flux entry driven by shielding current [8]. The SQUID placed in the middle of the structure has two parallel strips of $10 \mu \mathrm{m}$ width and a long hole perpendicular to the bicrystal line. The hole length and width are 60 and $20 \mu \mathrm{m}$, respectively. The $2 \mu \mathrm{m}$ wide bridges including grain boundary junctions were located at the end of the hole. The estimated inductance $L_{s}$ and effective area $A_{s}$ of the SQUID is about $70 \mathrm{pH}$ and $1960 \mu \mathrm{m}^{2}$, respectively. An effective area of the gradiometer $2 A_{\text {eff }}$ given by $A_{e f f}=\alpha A_{p} L_{s} / L_{p}$ can be calculated as $0.28 \mathrm{~mm}^{2}$.

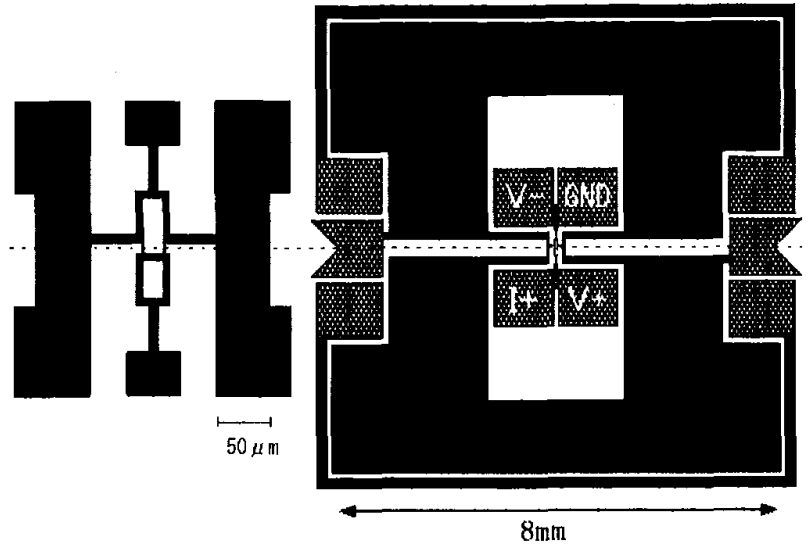

Fig. 5. Layout of a complete gradiometer and the inner part around the SQUID (The dashed line indicates the grain boundary).

\section{B. Gradiometric Response}

In planar parallel gradiometers the difference of the magnetic flux penetrating two pickup loops is converted into a current injected directly in a SQUID. If the pickup loops are equal in geometry, the flux measured by the SQUID is proportional to the spatial difference of the magnetic field. The characteristics of the gradiometer were measured at $77 \mathrm{~K}$ in a MSR. Fig. 6 shows the measured voltage-flux $(V-\Phi)$ characteristics under (a) symmetric and (b) anti-symmetric magnetic field. Defining the gradiometer balance as the ratio of magnetic fields needed to generate a flux quantum at the SQUID in Fig. 6 (a) and (b), we obtained a measured balance of $1 / 150(0.6 \%)$. This value is almost consistent with the ratio $A_{s} / 2 A_{\text {eff }}$ of $1 / 143(0.7 \%)$. Then, it is likely that the balance of the directly coupled gradiometer is limited by the sensitivity to homogeneous field of the SQUID itself.

When we drew the $V-\Phi$ curve under symmetric magnetic field larger than that for Fig. 6 (b), there were threshold fields at which voltage modulation disappeared. Fig. 7 shows $V$ - $\Phi$ characteristics when we applied flux more than $1.2 \Phi_{0}$ to the SQUID. Above the threshold field, the shielding current exceeded the critical current of the flux dam, and flux could not be transmitted to the SQUID. It is expected that flux dams can limit the shielding current induced by change of a field. If we reversed a direction of the field, the modulation voltage recovered immediately, probably due to high mobility of trapped flux in the grain boundary. We can observe some difference between the results for both direction of the field in Fig. 7. It is likely that this hysteresis originates from asymmetry of two pickup loops, i.e., only one pickup loop includes flux dams. The maximum threshold field at which flux dam would open was about $2.2 \mu \mathrm{T}$, and critical current of flux dam was $5 \mathrm{~mA}$.
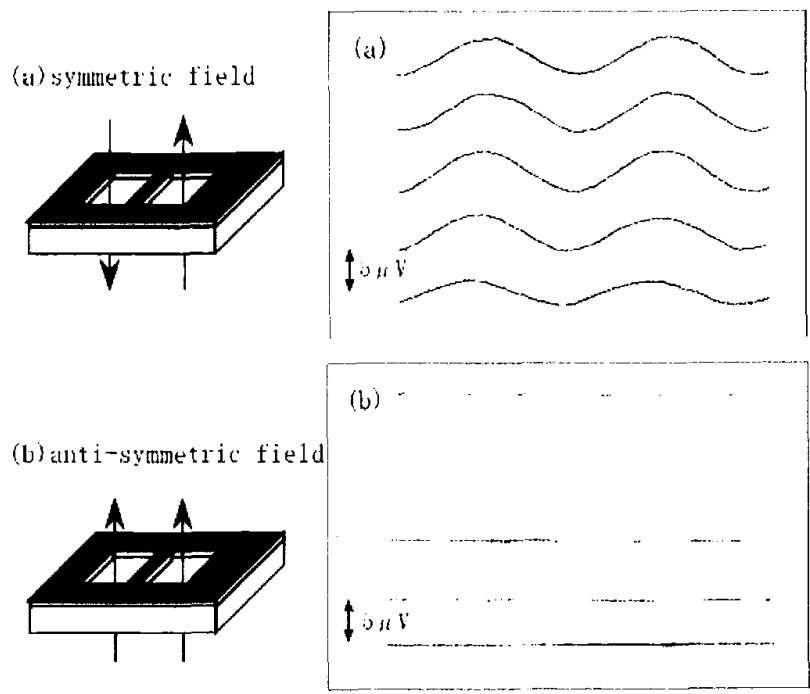

Fig. 6. V-Ф characteristics of the gradiometer under (a) symmetric and (b) anti-symmetric magnetic field. 


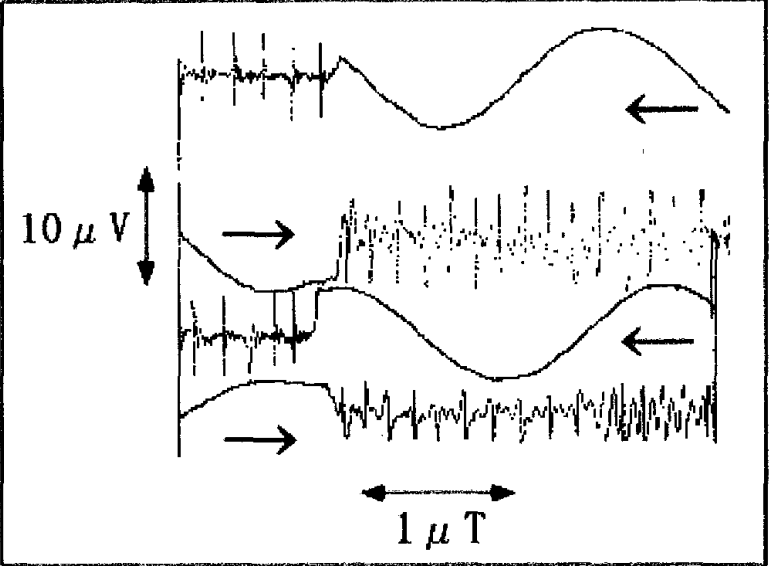

Fig. 7. V- $\Phi$ characteristics of the gradiometer under symmetric magnetic field larger than that in Fig. 6 (b).

\section{Noise Properties of Gradiometer}

Fig. 8 shows the noise spectra of the gradiometer, in FLL operation of static bias current, in an unshielded environment (outside a MSR) and in the MSR, respectively. The device worked stable even in laboratory environment without any shielding. While peaks arise at the frequencies of power lines and their harmonics, white noise level does not rise in unshielded operation. The increase of low frequency noise was observed at lower frequency region than a few $\mathrm{Hz}$ for unshielded case. At $1 \mathrm{~Hz}$, the flux noise was $200 \mu \Phi_{0} / \mathrm{Hz}^{1 / 2}$ which reduces to $20 \mu \Phi_{0} / \mathrm{Hz}^{1 / 2}$ at $1 \mathrm{kHz}$. These level of flux noise correspond to gradient sensitivities $\mathrm{S}_{\mathrm{g}}{ }^{1 / 2}=\mathrm{S}_{\Phi}{ }^{1 / 2} /\left(2 b A_{\text {eff }}\right)$ of $10 \mathrm{pT} / \mathrm{cmHz}^{1 / 2}$ at $1 \mathrm{~Hz}$ and $1 \mathrm{pT} / \mathrm{cmHz}^{1 / 2}$ at $1 \mathrm{kHz}$.

\section{CONCLUSIONS}

High- $T_{c}$ planar SQUID magnetometers and first derivative gradiometers with directly-coupled pickup loops

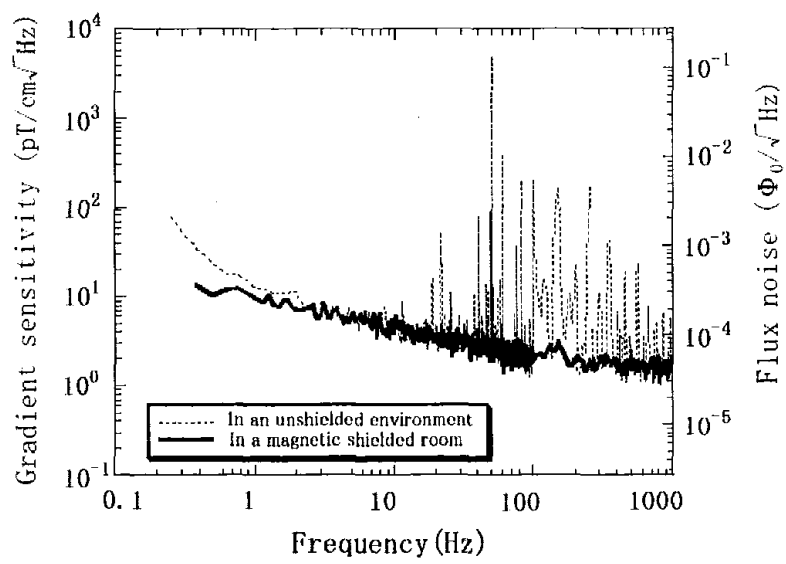

Fig. 8. Noise spectra of the dc SQUID gradiometer measured in an unshielded environment and in a MSR. were fabricated and characterized. From the viewpoint of the practical applications, special attention was paid to the operation in static magnetic field or in laboratory environment.

For the SQUID magnetometer, magnetic properties were investigated by applying a magnetic field $B_{0}$. We found an increasing low frequency noise with cooling fields $B_{0}$ larger than $1.5 \mu \mathrm{T}$, consisting with the threshold field estimated from the maximum structural width. A magnetic field noise level was about $500 \mathrm{fT} / \mathrm{Hz}^{1 / 2}$ at $10 \mathrm{~Hz}$. Measurements of MCG signals demonstrate the suitability of this magnetometer for biomagnetic applications in a moderate MSR.

For the gradiometer, operation without any shielding can be confirmed. Operation of flux dams included within the pickup loop was observed. The performance obtained was a field gradient resolution of about $1 \mathrm{pT} / \mathrm{cmHz}^{1 / 2}$ at $1 \mathrm{kHz}$ and $10 \mathrm{pT} / \mathrm{cmHz}^{1 / 2}$ at $1 \mathrm{~Hz}$, with a baseline of $4 \mathrm{~mm}$. The imbalance of the gradiometer was about $0.7 \%$, limited by the sensitivity to uniform field of the SQUID itself.

\section{REFERENCES}

[1] M. J. Ferrari, M. Johnson, F. C. Wellstood, J. J. Kingston, T. J. Shaw, and J. Clarke, "Magnetic flux noise in copper oxide superconductors," J. Low Temp. Phys., vol. 94, pp. 15, 1994.

[2] E. Dantsker, S. Tanaka, P. A. Nilsson, R. Kleiner, and J. Clarke, "Reduction of $1 / f$ noise in high- $T_{c}$ de superconducting quantum interference devices cooled in an ambient magnetic field," Appl. Phys. Lett, vol. 69, pp. 4099, 1996.

[3] E. Dantsker, S. Tanaka, and J. Clarke, "SQUIDs with slots and holes: Low $1 / f$ noise in ambient magnetic field," Appl. Phys. Lett., vol. 70, pp. 2037, 1997.

[4] S. Knappe, D. Drung, T. Schurig, H. Koch, M. Klinger, and J. Hinken, "A planar $\mathrm{YBa}_{2} \mathrm{Cu}_{3} \mathrm{O}_{7-\delta}$ thin-film gradiometer at $77 \mathrm{~K}$," Cryogenics, Vol. 32, pp.881, 1992.

[5] V. Zakosarenko, F. Schmidl, H. Schneidewind, L. Dorrer, and P. Seidel, "Thin-film de SQUID gradiometer using a single $\mathrm{YBa}_{2} \mathrm{Cu}_{3} \mathrm{O}_{7 . \delta}$ layer," Appl. Phys. Lett., vol. 65, pp. 779, 1994.

[6] C. Carr, A. Eulenburg, Ed Romans, C. M. Pegrum, and G. B. Donaldson, "Planar SQUID gradiometers fabricated on $24^{\circ}$ and $30^{\circ}$ $\mathrm{SrTiO}_{3}$ bicrystals," IEEE Trans. Appl. Superconductivity, vol. 9, pp. $3105,1999$.

[7] R. H. Koch, J. Z. Sun, V. Foglietti, and W. J. Gallagher, "Flux dam, a method to reduce extra low frequency noise when a superconducting magnetometer is exposed to a magnetic field," Appl. Phys. Lett., vol. 67, pp. $709,1995$.

[8] E. Maruyama, S. Kuriki, K. Yokosawa, R. S. Ahmad, D. Suzuki, and M. Koyanagi, "Flux coupling in the direct-coupled high-Tc superconducting quantum interference devices," Jpn. J. Appl. Phys., vol. 37, pp. L722, 1998. 